\title{
Norois
}

Environnement, aménagement, société

$245 \mid 2017$

Adapter les territoires aux changements climatiques : transition urbanistique et aménagement de l'espace

\section{Introduction. Adapter les territoires aux changements climatiques : transition urbanistique et aménagement de l'espace}

René-Paul Desse, Alain François, Mario Holvoet et Jérôme Sawtschuk

\section{OpenEdition}

\section{Journals}

Édition électronique

URL : http://journals.openedition.org/norois/6201

DOI : 10.4000/norois.6201

ISBN : 78-2-7535-7465-6

ISSN : $1760-8546$

Éditeur

Presses universitaires de Rennes

Édition imprimée

Date de publication : 31 décembre 2017

Pagination : 7-13

ISBN : 978-2-7535-7456-4

ISSN : 0029-182X

Référence électronique

René-Paul Desse, Alain François, Mario Holvoet et Jérôme Sawtschuk, «Introduction. Adapter les territoires aux changements climatiques : transition urbanistique et aménagement de l'espace », Norois [En ligne], 245 | 2017, mis en ligne le 31 décembre 2017, consulté le 05 janvier 2021. URL: http://journals.openedition.org/norois/6201 ; DOI : https://doi.org/10.4000/norois.6201 


Presses
Universitaires
de Rennes
www.pur-editions.fr
Revue en ligne : http://norois.revues.org

\title{
Introduction - Adapter les territoires aux changements climatiques : transition urbanistique et aménagement de l'espace
}

\author{
Introduction - Adapt Territories to Climate Change: \\ Urbanistic Transition and Planning
}

\author{
René-Paul Desse ${ }^{a}$, Alain François ${ }^{b}$ et Jérôme SawstschuK ${ }^{c}$
}

\footnotetext{
Professeur d'urbanisme, Laboratoire de Géoarchitecture (EA 7462), université de Bretagne Occidentale.

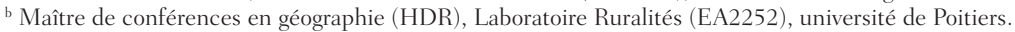

' Maître de conférences en urbanisme et aménagement, Laboratoire de Géoarchitecture (EA 7462), université de Bretagne Occidentale.
}

L'adaptation des territoires aux changements climatiques est devenue en quelques décennies un enjeu de société majeur et une réalité incontournable qui interroge les politiques publiques sur les manières de penser, d'habiter et d'aménager nos territoires urbains et ruraux à travers notamment la question des mobilités, l'usage et la gestion des ressources, le maintien et la protection de la biodiversité. Le Plan National d'Adaptation adopté en juillet 2011 en France soulignait combien « l'adaptation de notre territoire au changement climatique est devenue un enjeu majeur qui appelle une mobilisation nationale » et précisait que « Du point de vue sociétal, l'adaptation ne peut être dissociée des représentations de l'opinion publique sur le sujet controversé du changement climatique et des dispositions individuelles, collectives et institutionnelles à modifier des comportements pour faire face aux effets du changement climatique » (Le Treut, 2013).

Les sciences sociales ont fait de cette question un nouvel objet de recherche depuis les années 2000, comme en attestent les travaux du Tyndall Centre for Climate Change Research (Abramovitz et al., 2001 ; Adger, 2003; Brooks, 2003; Tompkin, 2004; Berkhout, 2004), ceux de l'International Institute for Sustenable Development et de l'International Union for Conservation of Nature. Parmi les publications francophones, figurent notamment les travaux du collectif dirigé par François Bertrand et Laurence Rocher sur Les territoires face aux changements climatiques. Une première génération d'initiatives locales paru en 2014, le dossier spécial de la Revue de Géographie des Pyrénées et du Sud-Ouest intitulé "Adaptations aux changements environnementaux et territoires » (2014), le numéro de Natures Sciences Sociétés sur «Les enjeux de la conférence de Paris. Penser autrement la question climatique » et l'ouvrage Environnement. Politiques publiques et pratiques locales sous la direction de Philippe Beringuier, Frédérique Blot, Bertrand Desailly et Mehdi Saqalli (2015). Enfin, depuis 1990, les Nations Unies ont publié les différents rapports du Groupe d'experts Intergouvernemental sur l'évolution du climat ou GIEC. Le cinquième rapport 
sous l'intitulé «Changements climatiques 2014, Impacts, vulnérabilité et adaptation » a sans doute été le document scientifique le plus important de cette dernière décennie, son contenu ayant servi de base à la préparation des COP (Conference Of the Parties) de la Convention-cadre des Nations unies sur les changements climatiques.

Une lecture attentive de la production scientifique parue depuis les années 2000 montre l'évolution des approches et des regards sur le sujet. Il semblerait que l'on ait privilégié à partir des années 1980 une lecture « hors sol» des questions relatives aux changements climatiques et environnementaux qui minorait l'importance des facteurs sociaux, politiques et économiques qui leur étaient attachés. Jacques Theys notait en effet la nécessité de changer la façon dont les scientifiques et les responsables politiques ont «enfermé » la problématique dans une lecture scientiste et climato-centrée, teintée d'un « exceptionnalisme climatique » qui a longtemps fait du réchauffement climatique un objet de recherche à part, autonome et indépendant, circonscrit à ses composantes physico-chimiques objectives, mesurables en termes d'émission de gaz à effet de serre et d'augmentation de la température atmosphérique. Ce qui a longtemps entravé et retardé la prise en main du problème (Theys, 2015).

Ainsi, depuis les années 2010, la problématique du changement climatique est réintroduite au centre d'un questionnement de plus grande envergure, circonstancié et complexifié, qui se décline en termes d'impacts, de vulnérabilité, d'adaptation et de résilience multiples et différenciées selon les territoires, les sociétés et l'évolution des modes de vie. Jean-Louis Tissier relevait déjà en 1992 combien « L'analyse de la notion de contrainte, ici climatique, mais qui pourrait être étendue aux éléments du milieu permet de souligner, d'affirmer le caractère totalement relatif de cette notion quand elle est introduite dans une géographie qui se donne comme science sociale. Ce milieu non seulement n'est pas stable, pérenne dans ses éléments physiques, mais il doit encore être apprécié en relation avec des choix économiques, voire politiques"(Tissier, 1992). Par ailleurs, dans l'introduction de son ouvrage Les risques climatiques paru en 2002, Denis Lamarre précisait combien il était difficile d'établir des corrélations certaines entre l'évolution des températures au cours du $\mathrm{Xx}^{\mathrm{e}}$ siècle et les catastrophes «specta- culaires » : « L'incertitude $[\ldots]$ reste très importante, et les travaux engagés posent nombre de questions relatives à la gestion de l'incertitude et du risque par la société face à leur environnement naturel et plus généralement à l'ensemble des questions relatives à la gestion de la question par les sociétés : le degré de risque toléré, la compréhension des mécanismes de prise de décision collective, la prévention et la gestion des catastrophes naturelles (à court et moyen termes) »(Lamarre, 2002). Dès lors, parmi les études proposées aujourd'hui en sciences sociales, les approches constructivistes entendent dépasser les seules dimensions physiques du climat pour (re) situer la problématique de l'adaptation aux changements climatiques à l'interface climat-société. Il s'agit de sortir d'une approche essentialiste des problématiques climatiques pour les réinsérer dans un champ de réflexion élargi et pluridisciplinaire (Dahan et Guillemot, 2015); comment les données physico-chimiques de l'atmosphère s'articulent-elles avec les composantes sociales, techniques, économiques, politiques, culturelles et symboliques qui participent à la construction de la relation que les sociétés entretiennent avec le climat et à ses évolutions contemporaines?

Au-delà de cette prise de conscience de ces problématiques déjà anciennes au sein des sciences de l'environnement et des sciences sociales, il nous semble important de remarquer leur diffusion lente mais continue dans le monde des acteurs de l'aménagement et de la planification régionale et urbaine. Depuis le Sommet de Rio, en 1992, jusqu'à la COP 21 qui s'est tenue à Paris en novembre 2015, ces différents sommets internationaux ont suscité des engagements de la part de la plupart des États concernant la réduction de leurs émissions de $\mathrm{CO}_{2}$. Même si la COP 21 n'a pas la même signification pour tous les pays, elle est le point de départ d'une prise de conscience des politiques. Une année plus tard, la COP 22, en novembre 2016 à Marrakech, puis celle Bonn en 2017, ont permis d'entrevoir les limites d'une telle vague de promesses de réduction des différentes émanations de gaz à l'échelle des États, faute de volonté politique de beaucoup de participants, faute aussi d'institutions onusiennes susceptibles de vérifier le déroulement dans le temps de ces hypothétiques réductions. Pour autant, quelques pays ont commencé ou poursuivi, selon leur degré de sensibilisation à la problématique des 
changements climatiques, à décliner les exigences de la COP 21 dans leurs politiques énergétiques, dans le développement de leurs infrastructures de transports ou dans leurs politiques urbanistiques.

Ainsi, un certain nombre de pays, notamment européens, ont rédigé des documents cadres présentant leurs actions susceptibles d'adapter leurs territoires aux changements climatiques. La France, à la suite du Grenelle de l'environnement, a élaboré un PNACC ou Plan National d'Adaptation au Changement Climatique pour les années 2011 2015. Avec l'approche de la COP 21, le pays organisateur se devait de produire un nouveau Plan qui court jusqu'en 2020. Cette gouvernance verticale, bien française, a été relayée par la plupart des régions et des départements, alors que dans le même temps les collectivités locales se devaient d'inscrire cette thématique dans leurs actions territoriales via l'Agenda 21, les SCoT puis tout dernièrement les PLUI.

Cette démarche descendante du haut de l'État vers la base s'est mise au service de l'adaptation aux changements climatiques sans que la plupart des acteurs en présence ne sache trop ce que recouvre ce « concept ». Une des caractéristiques fondamentales de l'adaptation pourrait être sa polysémie et son ambiguïté, rejoignant ainsi le rang des « notions enveloppes, de concepts éponges » (Richard, 2016).

La diversité des pistes de réflexion proposées aujourd'hui montre l'ampleur et la richesse des chantiers ouverts sur de nombreux fronts et à différentes échelles. Ainsi, la contribution de Guillaume Christen apporte un éclairage sociologique original et bien venu sur les contours protéiformes de la question de la transition énergétique actuellement en cours pour lutter contre les changements climatiques. À partir d'enquêtes de terrain réalisées à l'échelle de l'ancienne région Alsace, il nous enseigne que la transition vers d'autres sources d'énergie s'appuie sur une dynamique sociale qui interroge notre rapport à la nature, à la technique et au politique. Il note également l'importance des héritages historiques et en particulier le poids du nucléaire civil en France qui conditionne les possibilités de changement en cours.

D’autres champs de réflexion sont également proposés dont l'intérêt réside dans le fait qu'il n'est plus envisagé de penser l'adaptation à partir des impacts ou de l'évolution en cours du climat. On souhaite au contraire inverser la logique du raisonnement en proposant une réflexion sur et à partir des sociétés saisies par la menace climatique (Bertrand et Rocher, 2014). Par exemple, quelles recompositions sociales, économiques, paysagères et patrimoniales se jouent aujourd'hui autour de ce que certains appellent la «transition urbaine » ?

En effet, à l'échelle de la ville ou d'un quartier, l'urbanisme comme discipline se trouve au cœur de la problématique des changements climatiques. L'augmentation des vulnérabilités urbaines, attendues dans les prochaines décennies, comme une hausse des températures mais aussi les risques paroxysmiques comme les inondations sont à envisager en intra urbain. Urbanistes et spécialistes de l'ingénierie de la ville peuvent proposer une cartographie de la vulnérabilité de ces espaces en fonction de paramètres tels que l'intensité du dôme de chaleur, les effets d'ombrage, le vent. Néanmoins, une observation à une échelle géographique plus large permet d'intégrer les solidarités entre les territoires. S’il est prouvé que le centre d'une grande agglomération comme Paris peut générer un dôme de chaleur supérieur à $6{ }^{\circ} \mathrm{C}$ par rapport aux espaces plus agricoles de l'Île-de-France, l'inverse est aussi une réalité (Masson et al., 2013). Différentes recherches ont ainsi montré l'influence que peuvent avoir les sols et leurs couverts sur la météorologie et la qualité de l'air d'une région ou du moins d'une aire métropolitaine. La contribution de Mathieu Perrin, Nathalie de Noblet-Ducoudré, Susanna Strada et André Torre est, à cet égard, riche d'expériences dans la prise en compte des apports de la climatologie régionale dans les pratiques de planification. Partant de l'exemple de l'agglomération de Stuttgart, concernée par d'importants problèmes de pollution de l'air, en raison notamment d'une localisation en fond de vallée encaissée, les auteurs montrent que dès 1938, la Municipalité engage une réflexion sur le sujet. En 1948, une cartographie des couloirs canalisant l'air froid, et donc susceptible de venir rafraîchir et renouveler l'air pollué présent sur l'agglomération, permet une intégration de ces problématiques dans le nouveau plan d'urbanisme général. Plus récemment, il est envisagé de tirer profit d'une planification territoriale élargie, à l'échelle du Grand Paris, pour notamment faire face à l'îlot de chaleur urbain sur le cœur d'agglomération et ainsi réduire sa vulnérabilité dans un contexte de réchauffement 
global (Masson et al., 2013). Plusieurs exemples, notamment aux États-Unis, permettent d'envisager des interactions entre des modifications des usages des sols, sous la forme de nouvelles cultures, et le climat plus spécifiquement urbain. En effet, potentiellement capables d'atténuer certains évènements météorologiques extrêmes ou d'améliorer la qualité de l'air, les sols et leurs couverts sont rarement pris en compte dans la planification régionale.

Toujours à l'échelle régionale, les changements climatiques en cours peuvent être appréhendés sur des territoires fragiles, susceptibles d'être affectés par la remontée des eaux marines. L'équipe de Mathieu Le Dez nous propose ainsi une approche écogéomorphologique de l'estuaire de la Basse Loire. La modélisation des dynamiques de la végétation et des systèmes hydro-sédimentaires intègre aussi leurs impacts sur les activités socio-économiques anciennes comme l'élevage ou la pêche, mais aussi les activités portuaires de la Basse Loire avec ses terminaux et ses exigences d'avoir un chenal périodiquement dragué pour une navigation maritime jusqu’à Nantes.

Si l'élévation du niveau de la mer entraîne une salinisation croissante des prairies actuelles, les modifications de la pluviosité et de l'évapotranspiration sur l'ensemble du bassin hydrographique de la Loire susciteront une réduction des débits du fleuve. Les conséquences sur le transit des sédiments via l'augmentation du bouchon vaseux créeront de fortes modifications dans l'écosystème de l'estuaire avec des aspects positifs comme la remise en eau de certaines prairies au profit de certaines espèces d'oiseaux; et des aspects négatifs comme le recul de l'élevage ou la fragilisation de certains terre-pleins industriels. La présence de deux grandes agglomérations, aux deux extrémités de l'estuaire, a permis l'élaboration d'un SCoT de la Métropole Nantes Saint-Nazaire. Regroupant 61 communes, ce document de planification a le défaut de ne prendre en compte que la rive nord de la Basse Loire. Il pourrait néanmoins devenir un outil de réflexion intégrant ces changements dans un milieu estuarien soumis aux influences marines et fluviales; ce qu'il n'est pas pour l'instant.

En milieux urbains et périurbains, la transition urbanistique est en marche, souvent à base d'élaboration de documents de planification, plus rarement constituée de vraies politiques d'adaptation de la ville aux changements climatiques, mais presque toujours accompagnée d'un marketing territorial impressionnant.

La première phase de cette transition s'est amorcée à la fin des années 1980 pour se déployer dans les années 1990 par l'élaboration de normes, de labels et de certifications ayant trait aux économies d'énergie. Ainsi, les labels HQE qui ont été définis dans les années 1990-1996, avant d'être périodiquement réactualisés. Il en va de même des écoquartiers. Les premières expériences en Allemagne ou dans les pays scandinaves ont permis de faire connaître ce nouveau concept. En France, à la suite du Grenelle de l'environnement promulgué en 2009, le ministère du Logement et de l'Habitat durable a lancé leur labellisation au début des années 2010.

La deuxième phase est caractérisée par l'apparition d'une panoplie d'outils de planification. En France, la loi Grenelle 2 oblige les collectivités locales de plus de 50000 habitants à élaborer un plan climat-énergie territorial (PCET) ${ }^{1}$. Il s'agit d'atténuer et de réduire les émissions de gaz à effet de serre et d'adapter le territoire aux changements climatiques. Pour la plupart de ces PCET, les priorités sont d'abord énergétiques comme moderniser les réseaux de chaleur, moderniser l'éclairage public, proposer des alternatives aux déplacements motorisés. Les préoccupations proprement urbanistiques, notamment liées à l'étalement urbain et à l'accélération de la périurbanisation ne sont pris que récemment en compte. Ainsi, les PLU facteur 4 intègrent à la fois le Plan de Déplacements Urbains (PDU), le Programme Local d'Habitat (PLH) et le Plan Climat Energie Territorial $(\mathrm{PCET})^{2}$. Cette approche globalisante de la nouvelle planification urbaine locale propose pour la première fois un document d'urbanisme opposable aux tiers intégrant des problématiques d'étalement urbain et de consommation d'espaces agricoles par l'habitat et les activités économiques. Il est encore trop tôt pour pouvoir établir un premier bilan. Par contre, se pose déjà le problème de l'échelle pertinente. En devenant obligatoires, à partir de mars 2017 suite à la loi ALUR de 2016, les PLU intercommunaux constituent une nette amé-

\footnotetext{
1. Depuis 28 juin 2016, par décret, la mise en œuvre d'un Plan Climat Air Energie Territorial est obligatoire pour les EPCI de plus de 50000 habitants à partir du $1^{\text {er }}$ janvier 2017 et au plus tard le 31 décembre 2018 pour les EPCI de plus de 20000 habitants.

2. L'expression facteur 4 désigne l'engagement à diviser les émissions de Gaz à effet de serre d'un territoire donné entre 1990 et 2050.
} 
lioration dans le choix d'un périmètre opérationnel pertinent. Pourtant, les PLU des grandes agglomérations n'englobent pas forcément l'ensemble de l'aire urbaine, et de ce fait, une partie de la régulation foncière est inefficace, permettant la poursuite d'un éparpillement périurbain.

Les présentations qui suivent abordent quelquesunes de ces thématiques liées à l'adaptation des espaces urbains aux changements climatiques proposant pour certaines de nouvelles formes urbaines, pour d'autres une réflexion sur les mobilités ou les politiques énergétiques.

Ainsi, Jean-Louis Maupu et Vaclav Stransky proposent un nouvel agencement des lieux et des circulations pour lutter contre le réchauffement climatique. Cette vision d'une nouvelle configuration urbaine axée sur un réseau de transports en commun et de fret en boucle permet de proposer une autre alternative à l'étalement urbain des périphéries des villes et à son prolongement, l'éparpillement périurbain. Cette nouvelle organisation se profile déjà depuis plusieurs décennies dans les villes moyennes et les métropoles avec des concentrations d'activités tertiaires de toutes sortes qui font que ces espaces connectés aux rocades deviennent des centralités à part entière concentrant massivement l'emploi ${ }^{3}$. Mais ces nouveaux espaces périphériques fortement consommateurs de terres agricoles ne sont accessibles qu'en voiture, rarement par les transports en commun. Jean-Louis Maupu et Vaclav Stransky imaginent de nouvelles configurations spatiales qui constitueraient de vrais morceaux de ville aux fonctions plurielles. Les fortes densités de population dessineraient un chapelet de quartiers, ce qui permettrait l'usage massif du chemin de fer ou d'une façon générale de transports en commun en site propre. Réduisant fortement le mouvement en tous sens des mobilités automobiles, ces infrastructures en boucle aideraient chacun à lutter contre les changements climatiques. De cette vision de la ville que certains pourraient percevoir comme utopique, nous préférons la comprendre comme une contribution à un nouvel urbanisme en marche. À la suite de Michel Lussault (2013) qui caractérise l'urbain

3. Ainsi 60 à $70 \%$ des parts de marché du commerce de détail se trouvent localisés dans ces périphéries. Avec quelques décennies de retard sur les métropoles, les villes moyennes voient actuellement se multiplier les parcs de bureaux périphériques. Dernier avatar de cet abandon des espaces centraux, les cabinets médicaux à la recherche de localisation accessibles aux habitants de la périphérie et du périurbain. contemporain comme « une configuration désagglomérée, avec autant de vides que de pleins », nous pouvons regretter que l'urbanisme et notamment l'urbanisme opérationnel n'ait pas de réponses voire peu de questionnements en la matière, notamment face aux changements climatiques.

Cette approche globalisante de la ville concevant l'évolution de la trame urbaine en fonction des mobilités durables, nous la retrouvons dans l'analyse de Frédéric Héran. Alors que la plupart des méthodes utilisées pour appréhender les déplacements urbains sont monomodales ou bimodales, il nous propose d'y intégrer la concurrence entre les modes de déplacements, y compris entre les mobilités actives (marche, vélo) et les mobilités en transports en commun, pour in fine être capable de proposer une offre cohérente en milieu urbain dense, la modération de la circulation automobile apparaissant comme la clé de voûte d'une politique de déplacement plus équilibrée.

La présentation de la mise en place d'un transport collectif en site propre à Douala nous permet de nous décentrer de nos préoccupations de pays développés pour aborder l'exemple d'une ville millionnaire où les déplacements se font massivement en mototaxis et en voitures. Face à un étalement urbain de plus en plus important, suscité notamment par l'habitat informel, la mise en place de transports en commun n'est pas simple. Claudia Manto Teignegou et ses collègues analysent la mise en œuvre d'un Bus Rapid Transit à l'échelle de l'agglomération. Si les conséquences positives d'une telle infrastructure sont reconnues par l'ensemble des acteurs, en termes de réduction des émissions des gaz à effet de serre, la mise en ouvre d'une telle infrastructure tarde faute d'une autorité compétente à l'échelle de l'agglomération. L'éparpillement des responsabilités tant au niveau de la planification que de la maîtrise opérationnelle du futur chantier suscite des blocages, d'autant plus que les aires de compétences des différentes institutions publiques s'enchevêtrent (ministères, communauté urbaine) et ne recouvrent pas forcément l'ensemble du territoire de l'agglomération. Cette difficulté de concevoir la problématique des transports collectifs à la bonne échelle est commune à l'ensemble des grandes agglomérations et suscite de nombreux débats chez les élus des collectivités locales concernées, chacun essayant de conserver son pré carré, au détriment 
d'une gouvernance urbaine plus efficace à l'échelle métropolitaine.

Une attention est désormais également portée à l'acceptation sociale des dispositifs et des politiques mis en œuvre, considérant qu'il s'agit là d'un élément important pour apprécier l'efficacité et la pérennité des actions entreprises. Par exemple, le texte de François Bertrand et de Timothée Fouqueray analyse les modalités de gestion des ressources naturelles au sein d'un Parc Naturel Régional (PNR), celui de Loire-Anjou-Touraine, exposé aux effets du changement climatique. Les auteurs insistent sur la nécessité de réinterroger les pratiques et les référentiels des politiques locales de conservation de la biodiversité. Comment l'adaptation aux changements climatiques (ACC) contribue-t-elle à l'évolution des politiques et des pratiques locales en faveur de la biodiversité? La richesse du texte tient à l'analyse qui est proposée d'une expérimentation actuellement en cours dans le PNR Loire-Anjou-Touraine avec le double regard des sciences de l'aménagement et de la conservation. Une évaluation de l'acceptabilité et des implications socio-écologiques de cette " démarche-test » autour de l'adaptation est faite ainsi que de ses effets en termes de gouvernance. À partir de l'analyse d'un cas concret, la prise en compte de l'adaptation aux changements climatiques invite, selon les auteurs, à re-questionner les objectifs mêmes des politiques actuelles de conservation de la biodiversité, leurs référentiels d'action et leurs modalités de mise en œuvre.

Enfin, comment les politiques d'adaptation participent à la réorganisation des jeux de pouvoirs en ville comme à la campagne en matière de lutte contre les inégalités, de régulation des conflits, d'évolution des modes de vie etc. ? Parce que l'évaluation des stratégies d'adaptation n'est plus réductible à la seule gestion des écosystèmes, parce que l'environnement est devenu un objet et un instrument des politiques publiques, enfin parce que l'incertitude qui prévaut porte encore sur l'efficacité des stratégies d'adaptation à mettre en œuvre pour anticiper, limiter ou lutter contre les impacts climatiques indésirables en termes de développement, la question appelle une approche renouvelée, globale et intégrée, à inventer et à interroger devant la complexité des enjeux et la diversité des acteurs concernés. La contribution de Guillaume Simonet revient sur quelques aspects de cette complexité et souligne notamment la difficulté de traduire sur le terrain des mesures dites «d'adaptation aux changements climatiques ». Il relève l'inadéquation entre la construction théorique de la notion d'adaptation et la complexité d'une réalité à laquelle les acteurs sont confrontés sur le terrain. L'auteur fini par conclure que pour répondre aux changements climatiques, l'action publique territoriale gagnerait à être abordée de manière davantage systémique autour des enjeux environnementaux.

Ainsi, la question de l'adaptation des territoires aux changements climatiques suscite de nombreuses interrogations qui obligent pour l'heure à la formulation d'hypothèses de travail prudentes et nécessairement provisoires. Quoi qu'il en soit, et malgré ses aspects multiformes et non définitifs, la question de l'adaptation des territoires au changement climatique apparaît comme un champ de réflexion riche et stimulant pour les sciences sociales.

Parmi les huit textes rassemblés dans ce numéro, ceux de Guillaume Christen, de Guillaume Simonet et de François Bertrand et Timothée Fouqueray sont issus d'un séminaire de recherche intitulé Territoires, environnement et biodiversité face aux changements climatiques : Impacts et adaptations organisé à Poitiers le 11 mai 2016 par la Communauté d'agglomération du Pays Châtelleraudais, l'association GEREPI et le laboratoire Ruralités (EA2252) de l'université de Poitiers ${ }^{4}$.

Ceux de Mathieu Le Dez, Jérôme Sawtschuk, Frédéric Bioret, Pierre Le Hir et de Régis Walther; de Mathieu Perrin, Nathalie de Noblet-Ducoudré, Susanna Strada et André Torre; de Frédéric Héran; de Jean-Louis Maupu et Vaclav Stransky; et de Claudia Manto Teignegou, André Nso Ngang et Joséphine Mireille Akoa Etoa sont issus du colloque Changements climatiques, aménagement de l'espace et transition urbanistique, organisé par le laboratoire de Géoachitecture (EA 7462, université de Bretagne Occidentale), les 5 et 6 octobre 2015.

\footnotetext{
4. Les travaux du laboratoire Ruralités (EA 2252) de l'université de Poitiers s'inscrivent dans le cadre du Contrat de Plan Etat-Région (CPER) INSECT 2015-2020 et du FEDER.
} 


\section{Bibliographie}

Abramovitz et al., 2001. Adapting to Climate Change: Natural Resource Management and Vulnerability Reduction, Background Paper to the Task Force on Climate Change, Adaptation and Vulnerable Communities, 37 p.

Adger W. N., 2003. Social Capital, Collective Action, and Adaptation to Climate Change, Economic Geography, oct., no 79 (4), p. 387-404.

Beringuier Ph., Blot F., Desailly B., Sagalli M., 2015. Environnement. Politiques publiques et pratiques locales, Paris, L'Harmattan.

Berkhout F., Hertin J., Arnell N., 2004. Business and Climate Change: Measuring and Enhancing Adaptive Capacity, Tyndall Centre for Climate Research an CSERGE, Norwich, The ADAPT project, $44 \mathrm{p}$.

Bertrand F., Rocher L., 2014. Les territoires face aux changements climatiques. Une première génération d'initiatives locales, Bruxelles, Peter Lang, coll. « EcoPolis », 269 p.

Bвоокs N., 2003. Vulnerability, risk and adaptation: A conceptual framework, Tyndall Centre for Climate Research an CSERGE, Norwich, Working paper n 38, 20 p.

Choay F., Merlin F., 2015. Dictionnaire de l'urbanisme et de l'aménagement, PUF, Coll. Quadrige.

Dahan A., Guillemot H., 2015. « Les relations entre science et politique dans le régime climatique : à la recherche d'un nouveau modèle d'expertise? ", in Nature, Sciences Sociétés, p. 6-18. [http://www.cairn.info/revue-natures-sciencessocietes-2015-Supp. 3-page-6].

Eliasson I., 2000. The use of climate knowledge in urban planning, in Landscape and Urban Planning n ${ }^{\circ} 48$, p. 31-44.

GIEC, 2013. Éléments physiques du climat, premier [http:// www.developpement-durable.gouv.fr/Rapports-d-evaluation.html] volume du cinquième rapport d'évaluation du groupe d'experts intergouvernemental sur l'évolution du climat (GIEC).
GIEC, 2014. Changements climatiques 2014 Incidences, adaptation et vulnérabilité. Contribution du Groupe de travail II au cinquième Rapport d'évaluation du Groupe d'experts intergouvernemental sur l'évolution du climat. [https://www.ipcc. ch/pdf/assessment-report/ar5/wg2/ar5_wgII_spm_fr.pdf].

Lamarre D., 2002. Les risques climatiques, Paris, Belin Sup, $224 \mathrm{p}$.

Le Treut H. (dir.), 2013, Les impacts du changement climatique en Aquitaine. Un état des lieux scientifique, Bordeaux, LGPA Éditions/Presses universitaires de Bordeaux.

Lussault M., 2013. L'avènement du monde. Essai sur l'habitation humaine de la Terre, Paris, Seuil, 304 p.

Masson V., Lion Y., Peter A., Pigeon G., Buyck J., Brun E., 2013. «Grand Paris » : Regional landscape change to adapt city to climate warming, Climatic Change, vol. 117, $\mathrm{n}^{\circ} 4$, p. 769-782.

Rebotier J., 2014. Adaptation aux changements environnementaux et territoires, Numéro thématique de la revue SudOuest Européen, n 36, p. 5-12.

Richard E., 2016. L'adaptation aux changements climatiques, les réponses de l'action publique territoriale, Rennes, Presses universitaires de Rennes, coll. «Espace et Territoires », 283 p.

Theys J., 2015. "Le climat : une question de temps », in Natures Sciences Sociétés, Supplément 3.

Tissier J.-L., 1992. La géographie dans le prisme de l'environnement (1970-1990), in Roвic M.-C. (dir.), Du milieu à l'environnement. Pratiques et représentations du rapport homme/nature depuis la Renaissance, Paris, Economica, p. 201-236.

Tompkins E. L., Adger W. N., 2004. Does adaptive management of natural resources enhance resilience to climate change?, Ecology and Society 9(2): 10 [http://www.ecologyandsociety.org/vol9/iss2/art10/]. 